\title{
Stage IIB Cervical Cancer FIGO 2018
}

National Cancer Institute

\section{Source}

National Cancer Institute. Stage IIB Cervical Cancer FIGO 2018. NCI Thesaurus. Code

C162238.

Cervical carcinoma with parametrial involvement but not up to the pelvic wall. (FIGO 2018) 